\title{
Ultrasonography-assisted videomediastinoscopy in superior vena cava obstruction
}

\author{
Eugenio Pompeo, MD, Federico Tacconi, MD, Mario Marino, MD,
} Antonio Gatti, MD, and Tommaso Claudio Mineo, MD, Rome, Italy

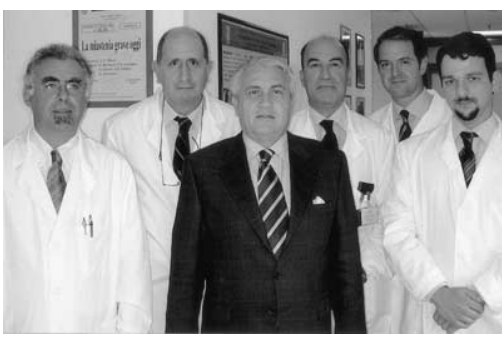

M. Marino, Sabato, C. Mineo, A. Gatti, E. Pompeo, F. Tacconi (left to right)

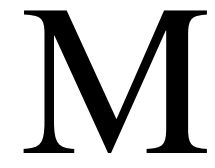
ediastinoscopy has been advocated as the most reliable technique to obtain rapid histologic diagnosis in superior vena cava obstruction. ${ }^{1,2}$ Nonetheless, iatrogenic injuries eventually requiring salvage sternotomy are reported with a low but constant frequency. ${ }^{2}$ To reduce the risks of major complications and increase diagnostic yield, we have integrated videomediastinoscopy with ultrasonographic assistance in patients with superior vena cava obstruction.

\section{Patients and Methods}

Between January 2002 and March 2005, we performed ultrasonographyassisted videomediastinoscopy (UAM) in 12 patients with superior vena cava obstruction. The major sign of presentation was neck swelling in all patients. Other symptoms and signs included dyspnea in 8 patients, weight loss in 4 patients, and hemoptysis in 1 patient.

UAM was always performed with the patient placed in a supine decubitus position and with the head and truncus elevated. The operative field was widely prepared as for a median sternotomy. General anesthesia with single-lumen armed endotracheal intubation was preferred.

Videomediastinoscopy (Richard Wolf, Knittlingen, Germany) was performed in the usual manner apart from a more careful dissection and accurate hemostasis. Once dissected free of the pretracheal space, the area targeted for biopsy was widely visualized. The operative field was then filled with saline solution before performing ultrasonography to reduce air-related artifacts. A miniaturized, 12-MHz, flexible ultrasonographic probe was inserted through the mediastinoscope and placed in proximity of the superior vena cava, the aorta, the right pulmonary artery, and the subcarinal space. The flexible probe was housed in a metallic

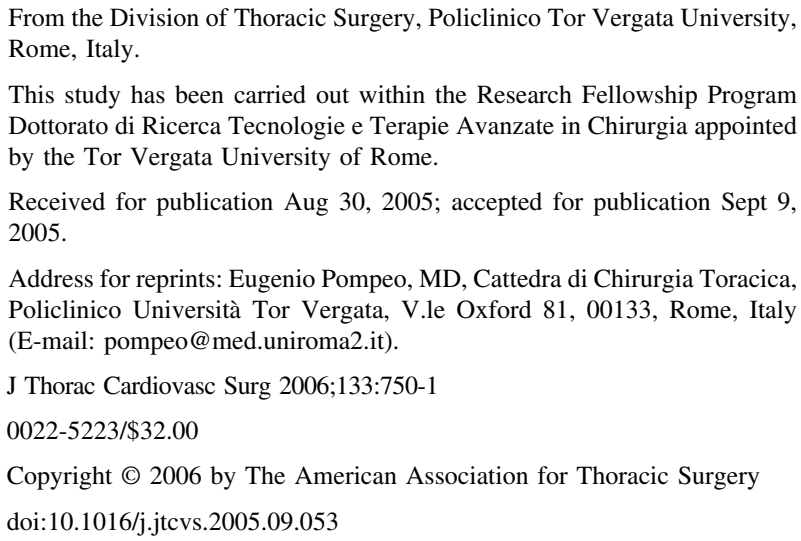

cannula to obviate its busy motion, leaving the probe tip protruding for about $2 \mathrm{~cm}$ (Figure 1). UAM offered a clear cross-sectional image (Figure 2) of the central vessels and neoplastic tissue, as well as their relationships. It also allowed us to discriminate primary tumor tissue from lymphoid tissue. In fact, the former has a hyperechoic dishomogeneous pattern, whereas isolated enlarged lymph nodes usually show a hypoechoic pattern with round shape and sharp borders. During endosonographic examination, the operative field was maintained clean from blood and fat drops, which can disturb echocardiographic imaging.

Because a cross-sectional ultrasonographic image resembles the axial computed tomographic scan, an endoscopic forceps (Figure 1) is introduced coaxially to the probe, and multiple biopsy specimens can be safely obtained while remaining distant from the vena cava. The mean number of biopsy specimens per patient was $4 \pm 2$ and ranged from 3 to 6 . The mean duration of the procedure was $33 \pm 12$ minutes and ranged from 22 to 40 minutes. There was no operative mortality and morbidity in this series.

At pathologic examination, a histologic diagnosis was achieved in all instances. Seven patients had lung cancer, 3 had nonHodgkin's lymphoma, and 1 had an invasive thymoma.

\section{Discussion}

Mediastinoscopy is a safe and effective tool in the diagnosis of superior vena cava obstruction. ${ }^{1}$ Nonetheless, iatrogenic lesions of the vena cava, innominate artery, azygos vein, trachea, and recur-

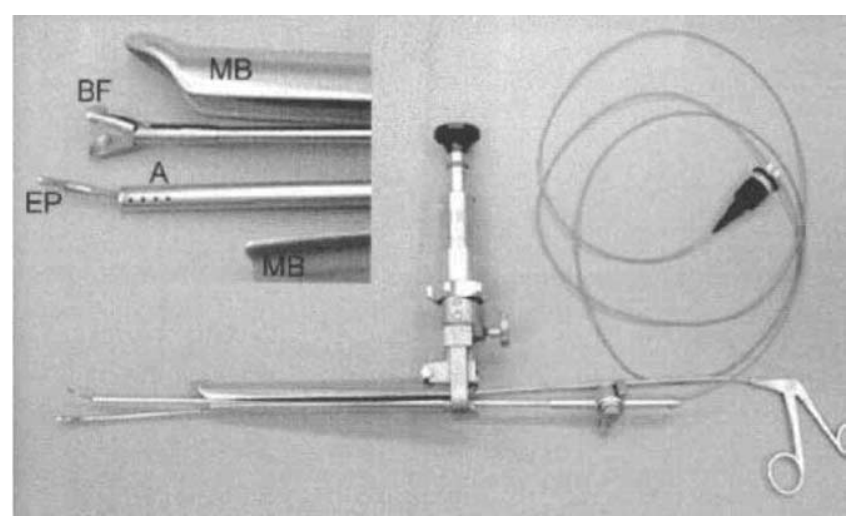

Figure 1. Instrumentation for ultrasonography-assisted videomediastinoscopy. A, Aspirator; EP, echographic probe tip; $B F$, biopsy forceps; $M B$, mediastinoscope branches. 

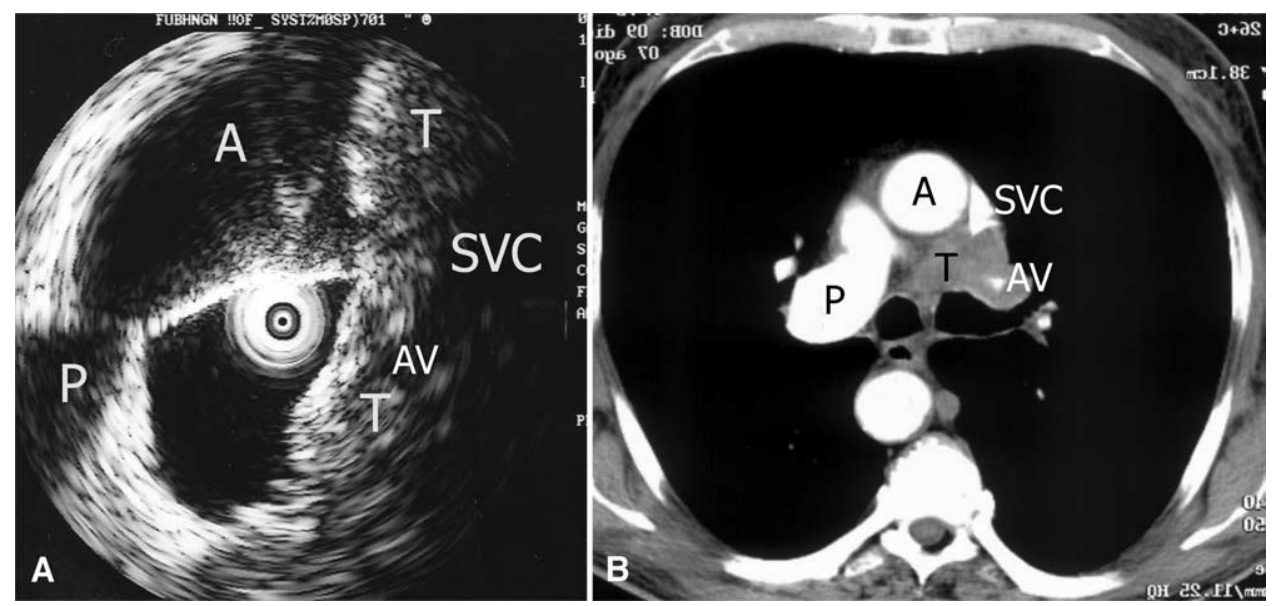

Figure 2. Left, A cross-sectional ultrasound image showing the tumor $(T)$, ascending aorta $(A)$, pulmonary artery $(P)$, superior vena cava (SVC), and azygos vein (AV). Right, The echographic image resembles the inverted tomographic scan and offers a real-time view of the tumor tissue and its relationships with neighboring structures. Abbreviations are as above.

rent nerve have been reported in large series. Furthermore, common carotid artery rupture requiring repair during extracorporeal circulation has also been reported. ${ }^{3}$ The introduction of video assistance has resulted in a significant advance in this field because of improved ergonomics and magnified vision.

Thus far, the combined use of mediastinoscopy with ultrasonography has been anecdotally reported in lung cancer staging. In 1995, Nakano and colleagues ${ }^{4}$ first used a conventional ultrasonographic probe to better assess mediastinal lymph node involvement. More recently, Hurtgen and associates ${ }^{5}$ have used a similar approach to ascertain resectability in a small series of patients with centrally located tumors. However, in both studies ultrasonographic exploration was limited to sole morphologic assessment, and no data regarding histologic findings were presented. Instead, the availability of a miniaturized echocardiographic probe has allowed us to use it coaxially with biopsy forceps and perform UAM for diagnostic aims. Although diagnostic yield of mediastinoscopy in superior vena cava obstruction as high as $100 \%$ has been reported in our previous series, ${ }^{1}$ we believe that UAM might lower the risks of major vascular complications, facilitating identification of the relationships existing between tumor tissue and major vessels. As a result, the surgeon is encouraged to obtain a greater number of samples, with a decreased likelihood of false-negative results. This is particularly useful in tumors involving the superior vena cava, in which neoplastic tissue can infiltrate the vessel wall, thus increasing the risk of vascular injury during mediastinoscopy.

In conclusion, although further investigation is warranted, in this small series, ultrasonography was useful to facilitate videomediastinoscopic biopsy in patients with superior vena cava obstruction.

\section{References}

1. Mineo TC, Ambrogi V. Mediastinoscopy in superior vena cava obstruction: analysis of 80 consecutive patients. Ann Thorac Surg. 1999;68: 223-6.

2. Jahangiri M, Goldstraw P. The role of mediastinoscopy in superior vena cava obstruction. Ann Thorac Surg. 1995;59:453-5.

3. Schmidli J, Vogt PR, Genoni M, Turina MI. Surgical management of severe common carotid artery injury after mediastinoscopy. Ann Thorac Surg. 2001;72:2107-9.

4. Nakano N, Nakahara K, Yasumitsu T, Kotake Y, Ikezoe J, Kawashima Y. Mediastinal ultrasonography for the assessment of mediastinal lymph node metastases in lung cancer patients. Surg Today. 1994;24: 106-11.

5. Hurtgen M, Metzler B, Friedel G, Toomes H. Mediastinoscopic ultrasonography (MUS). Eur J Cardiothorac Surg. 2004;26:842-4. 